\title{
Review Article \\ Nonalcoholic Fatty Liver Disease, Diabetes Mellitus and Cardiovascular Disease: Newer Data
}

\author{
A. N. Mavrogiannaki and I. N. Migdalis \\ 2nd Medical Department and Diabetes Center, NIMTS Hospital, 12 Monis Petraki, 11521 Athens, Greece \\ Correspondence should be addressed to A. N. Mavrogiannaki; anastasia_mavrogiannaki@yahoo.com
}

Received 9 March 2013; Accepted 12 March 2013

Academic Editor: Nikolaos Papanas

Copyright (C) 2013 A. N. Mavrogiannaki and I. N. Migdalis. This is an open access article distributed under the Creative Commons Attribution License, which permits unrestricted use, distribution, and reproduction in any medium, provided the original work is properly cited.

\begin{abstract}
Nonalcoholic fatty liver disease (NAFLD) is the most common, chronic liver disease worldwide. Within this spectrum, steatosis alone is apparently benign, while nonalcoholic steatohepatitis may progress to cirrhosis and hepatocellular carcinoma. NAFLD is strongly associated with obesity, dyslipidemia, type 2 diabetes mellitus, and cardiovascular disease. The pathogenesis of hepatic steatosis is not clearly known, but its main characteristics are considered insulin resistance, mitochondrial dysfunction, increased free fatty acids reflux from adipose tissue to the liver, hepatocyte lipotoxicity, stimulation of chronic necroinflammation, and fibrogenic response. With recent advances in technology, advanced imaging techniques provide important information for diagnosis. There is a significant research effort in developing noninvasive monitoring of disease progression to fibrosis and response to therapy with potential novel biomarkers, in order to facilitate diagnosis for the detection of advanced cirrhosis and to minimize the need of liver biopsy. The identification of NAFLD should be sought as part of the routine assessment of type 2 diabetics, as sought the microvascular complications and cardiovascular disease, because it is essential for the early diagnosis and proper intervention. Diet, exercise training, and weight loss provide significant clinical benefits and must be considered of first line for treating NAFLD.
\end{abstract}

\section{Introduction}

Nonalcoholic fatty liver disease (NAFLD) includes a wide spectrum of liver clinicopathologic conditions, ranging from pure fatty steatosis (fatty infiltration in $>5 \%$ of hepatocytes) which is apparently a benign condition to nonalcoholic steatohepatitis (NASH), which may progress to cirrhosis, liver failure, and hepatocellular carcinoma (HCC). It is characterized by excessive fat accumulation in the liver parenchyma of patients who have no history of alcohol abuse $(<20 \mathrm{~g}$ per day in men and $<10 \mathrm{~g}$ in women). NASH is characterized by biochemical evidence of hepatocellular damage (elevation of aminotransferase levels), histological findings of the type of alcoholic hepatitis (steatosis, lobular inflammatory cell infiltration, Mallory's hyaline, and fibrosis), and no other cause of liver damage.

NAFLD is the most common liver disease worldwide. Its reported prevalence varies, depending on the population of the study and using diagnostic criteria. In the general population exceeds $15 \%$, but it is far greater in the overweight, obese, and in subjects with type 2 diabetes (T2DM) as well type 1 diabetics [1-3]. Recent data indicate high prevalence in adolescents. The overall prevalence of NAFLD is reported $12.5 \%$, increasing to $23.0 \%$ in overweight/obese, higher in boys than in girls [4]. NAFLD is described in the $60 \%$ of the subjects with mixed hyperlipidemia, and in $83 \%$ of those with both mixed hyperlipidemia and an elevated serum alanine aminotransferase (ALT) [5].

NAFLD is strongly associated with T2DM and cardiovascular disease (CVD). It is characterized by insulin resistance and mitochondrial dysfunction [6]. Indeed, there is a gradual increase in the severity of insulin resistance in the range of NAFLD which may contribute to the evolution of liver damage. Also, is associated with an increased risk of kidney disease in subjects with multiple CVD risk factors and tends to be considered as an independent CVD marker [7]. Diabetes, dyslipidemia, hypertension and CVD coexist more frequently in individuals with NAFLD [8].

Health cost appears to be greater in NAFLD individuals than in general population. When were used data from the 
"Study of Health in Pomerania," Germany, to assess the relation of fatty liver disease to self-reported health care utilization and costs at baseline and 5 years, in a general population cohort study of 4310 adults aged 20 to 79 years at baseline, subjects with NAFLD and increased serum ALT levels, after controlling for comorbid condition, had 26\% higher overall health care costs [9].

\section{NAFLD and T2DM}

From various studies, the prevalence of NAFLD seems higher in type 2 diabetics than in general population, independent of glycemic control [10]. Type 2 diabetics have approximately $80 \%$ more fatty liver compared with nondiabetics matched for age and sex [11]. In a study of 2589 individuals from the community-based Framingham Heart Study, after multivariate adjustment for other fat depots (visceral adipose tissue, waist circumference, and body mass index (BMI)), fatty liver remained associated with diabetes, impaired fasting glucose, hypertension, metabolic syndrome, HDL cholesterol, triglycerides, and adiponectin levels (all $P<0.001$ ), whereas associations with systolic (SBP) and diastolic (DBP) blood pressure were attenuated $(P>0.05)[12]$.

There are studies which highlight diabetes as risk marker for NAFLD/NASH appearance. In a study of 458 Italian patients with histological proven NASH, diabetes was the most significant marker of NASH and fibrosis and in those with normal ALT [13]. Severe fibrosis was independently predicted by diabetes $(\mathrm{OR}=1.8 ; 95 \% \mathrm{CI}, 1.4-2.3)$ in the overall series and in those with normal ALT and insulin resistance according to homeostasis model assessment (HOMAIR) $(\mathrm{OR}=1.97 ; 95 \% \mathrm{CI}, 1.2-3.7)$ in patients with normal ALT [13]. In a cohort of 827 patients with NAFLD, advanced fibrosis was associated with insulin resistance [14].

Several studies have shown that NAFLD predicts the appearance of diabetes independently of conventional risk factors, as obesity, insulin resistance, and metabolic syndrome, suggesting that NAFLD could have a direct causal relationship with the appearance of diabetes, probably by promoting the insulin resistance [15]. It has been shown that increased liver enzymes predict T2DM independent of obesity [16]. In another study, metabolic changes (lipid, liver enzymes, blood pressure, and body weight) potentially associated with conversion to diabetes were investigated, it was found that in subjects who converted to new-onset diabetes, ALT $(P=0.0005)$ and triglycerides' $(P=0.030)$ concentrations are increased in absence of changes in body weight up to 18 months before the diabetes manifestation, but neither parameters increased significantly in nonconverters with high baseline glucose concentrations $(>6.1 \mathrm{mmol} / \mathrm{L})$ [17].

The poor controlled diabetes, also, promotes or worsens hepatic steatosis, thus feeding a vicious cycle that binds the two situations.

Therefore, hepatic steatosis, diabetes, and metabolic syndrome are part of the same disease process ultimately leading to increased cardiovascular morbidity and mortality risk.

\section{NAFLD and CVD}

Several prospective, epidemiological studies have shown that elevation of liver enzymes and ultrasonographic appearance of hepatic steatosis are predictors of CVD independent of conventional risk factors [18, 19]. Indeed, among 1221 apparently healthy subjects who were recruited from a health check-up program, NAFLD was a predictor of CVD independent of conventional risk factors (odds ratio 4.12, 95\% CI, 1.58 to $10.75, P=0.004$ ) and had central role in the cardiovascular risk of metabolic syndrome [19]. Metabolic syndrome was also independently associated with cardiovascular events, but simultaneous inclusion of NAFLD and metabolic syndrome in a multivariate model revealed that NAFLD but not metabolic syndrome retained a statistically significant correlation with CVD [19]. In a study of subjects with elevated ALT levels was found NAFLD and increased coronary heart disease (CHD), as assessed by Framingham risk score [20]. In another study, NAFLD subjects' survival was found lower compared with matched controls after a mean followup of 13.7 years [21]. Mortality was not increased in patients with steatosis, but it was found higher in NASH patients. These subjects more often died from cardiovascular $(P=0.04)$ and liver-related $(P=0.04)$ causes [21].

Subjects with NAFLD have significantly higher carotid artery intima-media thickness (IMT), a marker of subclinical atherosclerosis, comparing with those without fatty liver disease (mean IMT $=0.417 \mathrm{~mm}$ versus $0.395 \mathrm{~mm}, P<0.001$ ), impaired endothelial function, and lower concentrations of adiponectin $[4,22]$. IMT is strongly associated with degree of hepatic steatosis, necroinflammation, and fibrosis among NAFLD patients $(P<0.001$ for all $)$ [23]. Similarly, the severity of histological features of NAFLD independently predicted carotid IMT $(P<0.001)$ after adjustment for all confounders associated with the presence and severity of coronary atherosclerosis and cardiovascular disease [23]. These results suggest that the severity of liver histopathology among NAFLD patients is strongly associated with early carotid atherosclerosis, independent of classical risk factors, insulin resistance, and the presence of metabolic syndrome [23]. When the vasodilatory response of the brachial artery was assessed in response to ischemia (a test of endothelial function) as well as cardiovascular profile (10-year risk of coronary events) in 52 NAFLD cases and 82 age- and sexmatched controls, were endothelial dysfunction and increased risk of cardiovascular events in NAFLD subjects compared with controls observed [24].

It seems that NASH can predict a more atherogenic risk profile in a manner that is partly independent of the contribution of visceral adiposity. In a study, the differential contribution of NASH and visceral adiposity to nontraditional cardiovascular risk biomarkers in adult men was assessed [25]. 45 consecutive, overweight male patients with biopsyproven NASH, 45 overweight men without ultrasounddiagnosed hepatic steatosis and 45 healthy male volunteers were included. All participants were matched for age; NASH and overweight patients were also matched for BMI and visceral adiposity (as estimated by abdominal ultrasonography) [25]. Plasma concentrations of high-sensitivity C-reactive 
protein (hs-CRP), fibrinogen, and plasminogen activator inhibitor-1 (PAI-1) activity were found markedly lower in nonobese healthy volunteers, intermediate in overweight nonsteatotic patients, and the highest in overweight subjects with biopsy-proven NASH, after adjustment for age, BMI, smoking, plasma triglycerides, and insulin resistance [25]. Also, the highest concentrations of adiponectin were found in nonobese healthy subjects and the lower in those with biopsyproven NASH [25].

The relationship between hypertension and NAFLD has also been investigated. Higher prevalence of NAFLD in nonobese nondiabetic hypertensive patients with normal liver enzymes compared with adjusted nonhypertensive subjects. It has been found [26].

Abnormal left ventricular energy metabolism, fat accumulated in the epicardial area despite normal left ventricular morphological features, and systolic and diastolic functions, in nondiabetic young with fatty liver compared with nondiabetic matched for anthropometric features without fatty liver. It has also been reported [27]. It is interesting that, the addition of pioglitazone to insulin therapy, in type 2 diabetics, reduced myocardial and hepatic steatosis [28].

NAFLD is significantly associated with an increased CVD risk (odds ratio (OR) 1.84, 95\% CI, 1.4-2.1, $P<0.001$ ) among type 2 diabetics independent of classical risk factors, liver enzymes, or metabolic syndrome [29]. The independent association among NAFLD and CVD is supported by another large study following type 2 diabetics for 6.5 years [30]. Significant association of NAFLD with incident CVD (hazart ratio 1.96 (1.4-2.7), $P<0.001$ ) by adjustment for sex, age, smoking, diabetes duration, HbAlc, LDL-cholesterol and medications was found [30].

NAFLD in type 1 diabetics is associated with higher prevalence of CVD compared with non-NAFLD type 1 diabetics independently of age, sex, smoking, diabetes duration, HbA1c, LDL-cholesterol, HDL-cholesterol, triglycerides, SBP, and medication use (adjusted OR 7.36, 95\% CI, 1.60-34.3, $P<0.01)[3]$.

\section{Pathogenesis of NAFLD}

The pathogenesis of hepatic steatosis remains poorly understood but its main characteristics are considered insulin resistance and mitochondrial dysfunction [6]. If insulin resistance precedes NAFLD or the opposite remains unanswered. The source of stored hepatic fat results from dietary carbohydrates and fatty acids and the release of fat from adipocytes, by lipolysis or de novo hepatic lipogenesis [6]. An imbalance between the mechanisms may modify the rate of fat oxidation and fat removal from the liver. Defects in multiple levels may tip the metabolic balance towards hepatic fat accumulation: excessive substrate supply to the liver (i.e., glucose and fatty acids), intrahepatic imbalance between lipid synthesis and oxidation; inadequate export to peripheral tissues; and a combination of the above [31]. Many molecular defects at these different steps have been described in NAFLD.

4.1. Hepatic Insulin Resistance and NAFLD. Insulin resistance leads to hepatocyte steatosis by stimulation of insulin secretion and by increased lipolysis in adipose tissue, which increases circulating fatty acids. Increased uptake of circulating free fatty acids (FFA) by hepatocytes leads to mitochondrial beta-oxidation overload, with the consequent accumulation of fatty acids within hepatocytes. Fatty acids are substrates and inducers of the microsomal lipoxygenases cytochrome P-450 2E1 and 4A, resulting in the production of free oxygen radicals capable of inducing lipid peroxidation of hepatocyte membranes [32]. Hyperinsulinemia resulting from insulin resistance increases the synthesis of fatty acids in hepatocytes by increasing glycolysis and favors the accumulation of triglycerides within hepatocytes by decreasing hepatic production of apolipoprotein B-100. Reactive oxygen species (ROS) trigger lipid peroxidation, which causes cell death and releases malondialdehyde (MDA) and 4-hydroxynonena (HNE). MDA and HNE cause cell death; cross-link proteins, leading to the formation of Mallory's hyaline, and activate stellate cells promoting collagen synthesis. HNE has chemotactic activity for neutrophils, promoting tissue inflammation. ROS also induce the formation of cytokines tumor necrosis factor- $\alpha$ (TNF- $\alpha$ ), transforming growth factor $\beta$ (TGF- $\beta$ ) and interleukin-8. TNF- $\alpha$ and TGF- $\beta$ cause caspase activation and hepatocyte death. TGF- $\beta$ activates collagen synthesis by stellate cells and activates tissue transglutaminases, promoting the formation of Mallory's hyaline. The TNF- $\alpha$ induced by ROS further impairs the flow of electrons along the respiratory chain in mitochondria. Mitochondrial ROS cause expression of the Fas ligand (a death receptor member of TNFR family) in hepatocytes, which normally express the membrane receptor Fas. The Fas ligand on one hepatocyte can interact with Fas on another hepatocyte, causing fractional killing [32].

Elevated levels of triacylglycerol (TAG), diacylglycerol (DAG) and free cholesterol, NF- $\kappa \mathrm{B}$ signaling activation, mitochondrial dysfunction, and FFA toxic role it have reported $[6,31]$.

4.2. Adipose Tissue Insulin Resistance and NAFLD. Adipose tissue insulin resistance is associated with increased hepatic fat synthesis, regardless of obesity [15]. Adipocytes account for approximately $60 \%-70 \%$ of the FFA used for hepatic triglyceride synthesis and very low-density lipoprotein (VLDL) secretion [31]. Many factors that regulate VLDL metabolism may promote steatosis.

The adipocyte is a dynamic endocrine organ and nutrient sensor that tightly regulates energy supply. When nutrient supply exceeds adipose tissue adaptation, adipocyte hypertrophy and other poorly understood factors set off a pathologic adipocyte-macrophage crosstalk. The final result is adipocyte insulin resistance and the chronic release of FFA with toxic effects in distant tissues such as muscle, liver, and pancreatic $\beta$-cells as well as on the heart and vascular bed [15]. The precise series of events occurring is incompletely understood. Although the mechanisms by which the hepatic fat aggregation is associated with adipose tissue inflammation were not elucidated, macrophages infiltration may contribute to adipose tissue insulin resistance.

Excessive FFA availability promotes the accumulation of intramyocellular lipids and the formation of a variety of 
fat-derived potentially toxic lipid metabolites such as ceramide and DAG that activate the IKK/NF- $\kappa$ B pathway and cause insulin resistance. Inhibition of muscle insulin signaling and insulin resistance inhibits insulin action on adipose glucose uptake and lipid synthesis, further increasing the rate of FFA release into the circulation, and contributes to the development of insulin resistance and type 2 diabetes [15].

There is ample evidence that increases of plasma FFA concentrations cause hepatic insulin resistance, impair insulin signaling, and stimulate hepatic glucose production by driving both hepatic gluconeogenesis and glycogenolysis [15]. Induction of hepatic insulin resistance is following lipid infusion and correlates closely with increase in plasma FAA [15].

4.3. From Insulin Resistance to NASH. It is known that only $10 \%-25 \%$ of NAFLD subjects develop NASH [33]. Factors responsible for this evolution have been subject to extensive research but still remain incompletely understood. Still, data are fragmented and arise largely from rodents given the natural difficulties of assessing human liver tissue. One must keep in mind that there are significant metabolic/molecular differences between livers from humans and rodents and even between rodent species.

With these limitations in mind, in an effort to organize the current understanding of NAFLD and NASH, a framework is proposed on the progression from adipose tissue insulin resistance to NAFLD and NASH [31]. A prerequisite or "first step" for NASH appears to be adipose tissue insulin resistance, providing the necessary "lipotoxic environment" that ensures ample substrate supply to the liver (i.e., high FFA flux) and compensatory hyperinsulinemia that stimulates excessive hepatic triglyceride synthesis and the formation of toxic saturated fatty acids. The "second step" towards NASH is the development of hepatic steatosis and of a lipid pool from where lipid-derived toxic metabolites may activate inflammatory pathways. Dietary and genetic factors may condition the metabolic adaptation of the liver to this harmful environment. Compensated steatosis exacerbates hepatic insulin resistance, stimulates VLDL secretion, and increases mitochondrial beta-oxidation. If a new steady state is achieved, only benign steatosis and/or dyslipidemia (high triglyceride, low HDL-cholesterol) takes place. The "third step" for the progression from simple "bland" steatosis to active necroinflammation depends on the ability of the liver to adapt to longstanding triglyceride accumulation. If mitochondrial function cannot adapt to the increased FFA flux and respiratory oxidation collapses, lipid-derived toxic metabolites activate inflammatory pathways and hepatocyte lipotoxicity with stimulation of chronic necrosis and inflammation. Fibrosis is the final "fourth step," involving chronic activation of hepatic stellate cells in a yet poorly understood cross-talk of Kupffer cells with hepatocytes. The magnitude of the crosstalk between hepatocytes, macrophages, and hepatic stellate cells determines the degree of the fibrogenic response and potential progression to cirrhosis. In this setting, low plasma adiponectin levels are believed to promote steatosis and fibrosis by allowing unchecked triglycerides synthesis and activation of hepatic stellate cells, respectively.
The association between NAFLD, insulin resistance, and T2DM seems to be strong and partly due to genetic and environmental factors. The polymorphism of certain genes has been shown both in animal model and human studies that plays a significant role [34-36]. Diets rich in saturated fats, soft drinks, and meat and low in antioxidants, fish, and omega-3 fat are associated with development of NAFLD [37, 38].

\section{Recent Advances in Diagnosis of NAFLD}

The diagnosis of NAFLD/NASH is usually suspected in subjects with asymptomatic elevation of aminotransferase level, radiologic findings of fatty liver, or unexplained persistent hepatomegaly. The clinical diagnosis and liver tests have a poor predictive value with respect to histologic involvement. Imaging studies, although being of help in to determining the presence and amount of fatty infiltration of liver, cannot be used accurately determine the severity of liver damage. NAFLD is often diagnosed by a combination of clinical, laboratory and imaging data, but the clinical suspicion of NAFLD and its severity can only be confirmed with a liver biopsy [39]. Liver biopsy remains the best diagnostic tool for confirming NAFLD and evaluating necroinflammation/fibrosis, as well as the most sensitive and specific means of providing important prognostic information. Although liver biopsy is a relatively safe procedure when, performed by experienced clinicians, it has poor patient acceptance, it is not risk free and is difficult to be repeated.

Reliable and reproducible noninvasive methods for evaluating hepatocellular fat accumulation as well as the variable degree of hepatocyte necroinflammation (activity or grade of disease) and fibrosis (stage of disease), for frequent monitoring of disease progression, of treatment efficacy, and for prognosis assessment are strongly needed. With recent advances in technology, advanced imaging techniques (sonographic and magnetic elastography, magnetic spectroscopy) provide important information for diagnosis and usually diagnosis is based on these [40]. Several laboratory investigators try to identify potential novel biomarkers based on the current knowledge of the pathophysiologic mechanisms involved in NAFLD progression [41-43]. An ideal biomarker should be simple, reproducible, inexpensive, readily available, and accurate for a particular disease process. Potential rational targets for biomarkers development in NAFLD/NASH are based on the central role of inflammatory cytokines in the development of NAFLD, on the different oxidation products of several oxidation pathways, on mediators of fibrogenesis/fibrosis, on mediators/receptors involved in the hepatocyte apoptosis, and on breath biomarkers. Different mechanisms have been proposed including an increased production of reactive oxygen species and mitochondrial outer permeabilization, resulting in a cascade of events leading to inflammation (TNF- $\alpha$, adiponectin, C-reactive protein, IL-6, Resistin, and visfatin), hepatocellular apoptosis (Fas, circulating active caspase 3 ), fibrogenesis, and fibrosis (TGF$\beta$, tissue elasticity) [41]. It has evaluated the use of breath biomarkers in the study of NAFLD, such breath ethanol, ethane, and breath acetone [42]. Also, efforts attempts are 
made to identify noninvasive indicators of liver fibrosis by using routinely determined and easily available clinical and biochemical variables [44]. All these markers are under investigation and their clinical utility remains to be determined [41-44].

It is noted that with all above, diagnosis of significant fibrosis may be satisfactory, but that of steatosis and NASH without fibrosis is problematic.

\section{Therapy of NAFLD}

There is currently no established treatment for NAFLD or $\mathrm{NASH}$, although weight loss is recommended [39]. Several pharmaceutical interventions have been evaluated but none has been approved for general use. Most treatment studies have focused on subjects with NASH because of their potential to progress to fibrosis and cirrhosis; however, the findings have been limited by variations in treatment endpoints and a paucity of randomized, placebo-controlled, powerful and of sufficient duration trials.

Lifestyle changes, mostly focused on weight loss, have been demonstrated to improve liver aminotransferases and histological findings in obese with fatty liver [45]. In overweight or obese individuals with biopsy-proven NASH, weight reduction achieved through lifestyle intervention leads to improvements in liver histology [46]. The content of diet slimming is of no particular importance (advising to avoid alcohol), if lead to weight loss. However, although weight loss appears to be beneficial, rapid weight loss after gastroplasty has been associated with increased hepatitis despite reductions in steatosis on liver biopsy [47]. Weight loss agents had no significant effects compared with weight loss only [48]. In obese patients undergoing bariatric surgery, hepatic steatosis is decreased from $53 \%$ to $32 \%$, three months after surgery as overall mortality [49, 50]. Crosssectional investigations have shown an independent association between physical fitness and hepatic triglyceride concentration [51]. Regular exercise reduces hepatic and visceral lipids in previously sedentary obese individuals even in absence of weight loss [52].

Several pharmaceutical interventions have attempted to NAFLD/NASH, with limited benefit overall. Some studies have been performed with drugs acting cytoprotective or antioxidants or tumor necrosis factor antagonists or decreasing cytokines production, inhibitors of TGF- $\beta$, and semisynthetic agonists of receptor Farsenoid $\mathrm{X}$ with unsuccessful or moderate results [53-58]. In recent years, drugs that inhibit the system renin-angiotensin and $\alpha$-receptors with moderate biochemical and histological response are studied [59]. Sought treatment options for NASH, especially when is accompanied by fibrosis, but there are not large randomized trials.

Administration of lipid-lowering drugs has been evaluated in patients with NAFLD/NASH, but not in large prospective controlled trials and it has been associated with biochemical and histological improvement, but not all studies. The use of statins appears to be safe in patients monitored closely, to treat hyperlipidemia [39, 60]. The long-term ezetimibe therapy can lead to improvement in metabolic, biochemical, and histological abnormalities of NAFLD [61]. In small uncontrolled studies moderate or no benefit from use of fibrates has reported $[62,63]$.

The common metabolic disorders of T2DM and NAFLD can explain the greater success of drugs used to treat diabetes, with the most studies to target the use of drugs that improve insulin resistance. Use of metformin in NAFLD patients does not appear to help and is not recommended in nondiabetic NAFLD/NASH patients $[39,58]$. Clinical trials of pioglitazone have shown promising results (partial biochemical and histological efficacy) but the short-term effect and side effects may limit widespread acceptance [56]. At present, they are used only in clinical trials, while they can be used in type 2 diabetic patients with NAFLD/NASH. Intensive insulin therapy in type 2 diabetic patients with NAFLD/NASH appears to significantly decrease on steatosis [64]. GLP-1 receptors were detected on human hepatocytes and treatment with exendin4 quantitatively reduced triglyceride stores compared with control-treated cells [65]. The current preclinical evidence shows that GLP-1 analogs and DPP-4 inhibitors can improve hepatic steatosis independent of weight loss but is controversial whether the pancreatic-type GLP-1 receptor is present or responsible for conferring the GLP-1 signal in the hepatocyte [66].

In patients with NAFLD/NASH, all cardiovascular risk factors (obesity, hypertension, hyperglycemia, and hyperlipidemia) are treated $[39,67]$. The identification of NAFLD should be sought as part of assessment of diabetic patients for proper implementation of lifestyle and pharmaceutical interventions [68].

Liver transplantation is recommended in patients with decompensate cirrhosis due NASH as long as underlying comorbidities permit [32].

\section{Natural History: Prognosis}

NAFLD often follows a benign course but may leads to fibrosis, chirrosis due NASH and HCC. The evolution of fibrosis in NASH has been found in 25\%-33\% of the cases [69]. Factors favoring the evolution to cirrhosis are fibrosis, obesity (visceral), diabetes, and hypertension [70]. There is epidemiological evidence that $\mathrm{NASH}$ and cirrhosis are associated with increased HCC risk [71]. Mild steatosis is not associated with an increased risk mortality compared with general population [72]. In type 2 diabetics, presence of NAFLD is associated with increased total mortality, regardless of classic risk factors [73]. Diabetics with NAFLD have twice risk mortality compared with nondiabetics without NAFLD, with more common causes of death malignancy (33\% of death) and liver related complications (19\% of death) [73].

\section{Conclusions}

NAFLD is strongly associated with T2DM and CVD. Within this spectrum, steatosis alone is apparently benign, while nonalcoholic steatohepatitis may progress to cirrhosis and hepatocellular carcinoma. Its pathogenesis is complex and involves insulin resistance and mitochondrial dysfunction 
with increased FFA reflux from adipose tissue to the liver which play a key role in the chronic activation of inflammatory pathways and hepatocyte lipotoxicity with stimulation of chronic inflammation and necrosis. There is significant research effort in developing noninvasive monitoring of disease progression to fibrosis and response to therapy with potential novel biomarkers, which promise to facilitate diagnosis for the detection of advanced cirrhosis in order to minimize the need of liver biopsy, which are not used in clinical practice. The identification of NAFLD should be sought as part of routine assessment of diabetic patients, as sought the microvascular complications and CVD, because it is essential for the early diagnosis and proper implementation of lifestyle and pharmaceutical interventions. Diet, exercise, and weight loss provide significant clinical benefits and must be considered of first line for treating NAFLD/NASH.

\section{References}

[1] P. M. Gholam, D. P. Kotler, and L. J. Flancbaum, "Liver pathology in morbidly obese patients undergoing Roux-en-Y gastric bypass surgery," Obesity Surgery, vol. 12, no. 1, pp. 49-51, 2002.

[2] C. D. Williams, J. Stengel, M. I. Asike et al., "Prevalence of nonalcoholic fatty liver disease and nonalcoholic steatohepatitis among a largely middle-aged population utilizing ultrasound and liver biopsy: a prospective study," Gastroenterology, vol. 140, no. 1, pp. 124-131, 2011.

[3] G. Targher, L. Bertolini, R. Padovani et al., "Prevalence of nonalcoholic fatty liver disease and its association with cardiovascular disease in patients with type 1 diabetes," Journal of Hepatology, vol. 53, no. 4, pp. 713-718, 2010.

[4] C. A. Caserta, G. M. Pendino, A. Amante et al., "Cardiovascular risk factors, nonalcoholic fatty liver disease, and carotid artery intima-media thickness in an adolescent population in southern Italy," The American Journal of Epidemiology, vol. 171, no. 11, pp. 1195-1202, 2010.

[5] J. D. Browning, "Statins and hepatic steatosis: perspectives from the Dallas heart study," Hepatology, vol. 44, no. 2, pp. 466-471, 2006.

[6] H. Malhi and G. J. Gores, "Molecular mechanisms of lipotoxicity in nonalcoholic fatty liver disease," Seminars in Liver Disease, vol. 28, no. 4, pp. 360-369, 2008.

[7] G. Targher, C. P. Day, and E. Bonora, "Risk of cardiovascular disease in patients with nonalcoholic fatty liver disease," The New England Journal of Medicine, vol. 363, no. 14, pp. 1341-1350, 2010.

[8] G. Targher, F. Marra, and G. Marchesini, "Increased risk of cardiovascular disease in non-alcoholic fatty liver disease: causal effect or epiphenomenon?" Diabetologia, vol. 51, no. 11, pp. 19471953, 2008.

[9] S. E. Baumeister, H. Völzke, P. Marschall et al., "Impact of fatty liver disease on health care utilization and costs in a general population: a 5-year observation," Gastroenterology, vol.134, no. 1, pp. 85-94, 2008.

[10] N. C. Leite, G. F. Salles, A. L. E. Araujo, C. A. Villela-Nogueira, and C. R. L. Cardoso, "Prevalence and associated factors of nonalcoholic fatty liver disease in patients with type-2 diabetes mellitus," Liver International, vol. 29, no. 1, pp. 113-119, 2009.

[11] A. Kotronen, L. Juurinen, A. Hakkarainen et al., "Liver fat is increased in type 2 diabetic patients and underestimated by serum alanine aminotransferase compared with equally obese nondiabetic subjects," Diabetes Care, vol. 31, no. 1, pp. 165-169, 2008.

[12] E. K. Speliotes, J. M. Massaro, U. Hoffmann et al., "Fatty liver is associated with dyslipidemia and dysglycemia independent of visceral fat: the Framingham heart study," Hepatology, vol. 51, no. 6, pp. 1979-1987, 2010.

[13] A. L. Fracanzani, L. Valenti, E. Bugianesi et al., "Risk of severe liver disease in nonalcoholic fatty liver disease with normal aminotransferase levels: a role for insulin resistance and diabetes," Hepatology, vol. 48, no. 3, pp. 792-798, 2008.

[14] S. A. Harrison, D. Oliver, H. L. Arnold, S. Gogia, and B. A. Neuschwander-Tetri, "Development and validation of a simple NAFLD clinical scoring system for identifying patients without advanced disease," Gut, vol. 57, no. 10, pp. 1441-1447, 2008.

[15] K. Cusi, "The role of adipose tissue and lipotoxicity in the pathogenesis of type 2 diabetes," Current Diabetes Reports, vol. 10, no. 4, pp. 306-315, 2010.

[16] A. Kotronen and H. Yki-Järvinen, "Fatty liver: a novel component of the metabolic syndrome," Arteriosclerosis, Thrombosis, and Vascular Biology, vol. 28, no. 1, pp. 27-38, 2008.

[17] N. Sattar, A. McConnachie, I. Ford et al., "Serial metabolic measurements and conversion to type 2 diabetes in the West of Scotland Coronary Prevention Study: specific elevations in alanine aminotransferase and triglycerides suggest hepatic fat accumulation as a potential contributing factor," Diabetes, vol. 56, no. 4, pp. 984-991, 2007.

[18] R. K. Schindhelm, J. M. Dekker, G. Nijpels et al., "Alanine aminotransferase predicts coronary heart disease events: a 10year follow-up of the Hoorn Study," Atherosclerosis, vol. 191, no. 2, pp. 391-396, 2007.

[19] M. Hamaguchi, N. Takeda, C. Nagata et al., "Nonalcolholic fatty liver disease is a novel predictor cardiovascular disease," World Journal of Gastroenterology, vol. 13, no. 10, pp. 1579-1584, 2007.

[20] G. N. Ioannou, N. S. Weiss, E. J. Boyko, D. Mozaffarian, and S. P. Lee, "Elevated serum alanine aminotransferase activity and calculated risk of coronary heart disease in the United States," Hepatology, vol. 43, no. 5, pp. 1145-1151, 2006.

[21] M. Ekstedt, L. E. Franzén, U. L. Mathiesen et al., "Long-term follow-up of patients with NAFLD and elevated liver enzymes," Hepatology, vol. 44, no. 4, pp. 865-873, 2006.

[22] S. Treeprasertsuk, F. Lopez-Jimenez, and K. D. Lindor, "Nonalcoholic fatty liver disease and the coronary artery disease," Digestive Diseases and Sciences, vol. 56, no. 1, pp. 35-45, 2011.

[23] G. Targher, L. Bertolini, R. Padovani et al., "Relations between carotid artery wall thickness and liver histology in subjects with nonalcoholic fatty liver disease," Diabetes Care, vol. 29, no. 6, pp. 1325-1330, 2006.

[24] N. Villanova, S. Moscatiello, S. Ramilli et al., "Endothelial dysfunction and cardiovascular risk profile in nonalcoholic fatty liver disease," Hepatology, vol. 42, no. 2, pp. 473-480, 2005.

[25] G. Targher, L. Bertolini, S. Rodella et al., "NASH predicts plasma inflammatory biomarkers independently of visceral fat in men," Obesity, vol. 16, no. 6, pp. 1394-1399, 2008.

[26] G. Donati, B. Stagni, F. Piscaglia et al., "Increased prevalence of fatty liver in arterial hypertensive patients with normal liver enzymes: role of insulin resistance," Gut, vol. 53, no. 7, pp. 10201023, 2004.

[27] G. Perseghin, G. Lattuada, F. De Cobelli et al., "Increased mediastinal fat and impaired left ventricular energy metabolism in young men with newly found fatty liver," Hepatology, vol. 47, no. 1, pp. 51-58, 2008. 
[28] I. Zib, A. N. Jacob, I. Lingvay et al., "Effect of pioglitazone therapy on myocardial and hepatic steatosis in insulin-treated patients with type 2 diabetes," Journal of Investigative Medicine, vol. 55, no. 5, pp. 230-236, 2007.

[29] G. Targher, L. Bertolini, F. Poli et al., "Nonalcoholic fatty liver disease and risk of future cardiovascular events among type 2 diabetic patients," Diabetes, vol. 54, no. 12, pp. 3541-3546, 2005.

[30] G. Targher, L. Bertolini, S. Rodella et al., "Nonalcoholic fatty liver disease is independently associated with an increased incidence of cardiovascular events in type 2 diabetic patients," Diabetes Care, vol. 30, no. 8, pp. 2119-2121, 2007.

[31] K. Cusi, "Nonalcoholic fatty liver disease in type 2 diabetes mellitus," Current Opinion in Endocrinology, Diabetes and Obesity, vol. 16, no. 2, pp. 141-149, 2009.

[32] P. Angulo, "Medical progress: nonalcoholic fatty liver disease," The New England Journal of Medicine, vol. 346, no. 16, pp. 12211231, 2002.

[33] C. P. Day, "Natural history of NAFLD: remarkably benign in the absence of cirrhosis," Gastroenterology, vol. 129, no. 1, pp. 375378, 2005.

[34] K. F. Petersen, S. Dufour, A. Hariri et al., "Apolipoprotein C3 gene variants in nonalcoholic fatty liver disease," The New England Journal of Medicine, vol. 362, no. 12, pp. 1082-1089, 2010.

[35] N. M. W. de Alwis and C. P. Day, "Genetics of alcoholic liver disease and nonalcoholic fatty liver disease," Seminars in Liver Disease, vol. 27, no. 1, pp. 44-54, 2007.

[36] N. M. W. de Alwis and C. P. Day, "Genes and nonalcoholic fatty liver disease," Current Diabetes Reports, vol. 8, no. 2, pp. 156-163, 2008.

[37] G. Musso, R. Gambino, F. De Michieli et al., "Dietary habits and their relations to insulin resistance and postprandial lipemia in nonalcoholic steatohepatitis," Hepatology, vol. 37, no. 4, pp. 909916, 2003.

[38] S. Zelber-Sagi, D. Nitzan-Kaluski, R. Goldsmith et al., "Long term nutritional intake and the risk for non-alcoholic fatty liver disease (NAFLD): a population based study," Journal of Hepatology, vol. 47, no. 5, pp. 711-717, 2007.

[39] N. Chalasani, Z. Younossi, J. E. Lavine et al., "The diagnosis and management of non-alcoholic fatty liver disease: practice guideline by the American Association for the Study of Liver Diseases, American College of Gastroenterology, and the American Gastroenterological Association," The American Journal of Gastroenterology, vol. 107, no. 6, pp. 811-826, 2012.

[40] B. Taouli, R. L. Ehman, and S. B. Reeder, "Advanced MRI methods for assessment of chronic liver disease," The American Journal of Roentgenology, vol. 193, no. 1, pp. 14-27, 2009.

[41] A. Wieckowska, A. J. McCullough, and A. E. Feldstein, "Noninvasive diagnosis and monitoring of nonalcoholic steatohepatitis: present and future," Hepatology, vol. 46, no. 2, pp. 582-589, 2007.

[42] S. F. Solga, A. Alkhuraishe, K. Cope et al., "Breath biomarkers and non-alcoholic fatty liver disease: preliminary observations," Biomarkers, vol. 11, no. 2, pp. 174-183, 2006.

[43] S. C. Kalhan, L. Guo, J. Edmison et al., "Plasma metabolomic profile in nonalcoholic fatty liver disease," Metabolism, vol. 60, no. 3, pp. 404-413, 2011.

[44] P. Angulo, J. M. Hui, G. Marchesini et al., “The NAFLD fibrosis score: a noninvasive system that identifies liver fibrosis in patients with NAFLD," Hepatology, vol. 45, no. 4, pp. 846-854, 2007.
[45] T. Ueno, H. Sugawara, K. Sujaku et al., "Therapeutic effects of restricted diet and exercise in obese patients with fatty liver," Journal of Hepatology, vol. 27, no. 1, pp. 103-107, 1997.

[46] K. Promrat, D. E. Kleiner, H. M. Niemeier et al., "Randomized controlled trial testing the effects of weight loss on nonalcoholic steatohepatitis," Hepatology, vol. 51, no. 1, pp. 121-129, 2010.

[47] F. H. Luyckx, C. Desaive, A. Thiry et al., "Liver abnormalities in severely obese subjects: effect of drastic weight loss after gastroplasty," International Journal of Obesity, vol. 22, no. 3, pp. 222-226, 1998.

[48] S. A. Harrison, W. Fecht, E. M. Brunt, and B. A. NeuschwanderTetri, "Orlistat for overweight subjects with nonalcoholic steatohepatitis: a randomized, prospective trial," Hepatology, vol. 49, no. 1, pp. 80-86, 2009.

[49] J. R. van Werven, T. C. M. A. Schreuder, E. O. Aarts et al., "Hepatic steatosis in morbidly obese patients undergoing gastric bypass surgery: assessment with open-system $1 \mathrm{H}-\mathrm{MR}$ spectroscopy," The American Journal of Roentgenology, vol. 196, no. 6, pp. W736-W742, 2011.

[50] L. Sjöström, K. Narbro, C. D. Sjöström et al., "Effects of bariatric surgery on mortality in Swedish obese subjects," The New England Journal of Medicine, vol. 357, no. 8, pp. 741-752, 2007.

[51] J. B. Krasnoff, P. L. Painter, J. P. Wallace, N. M. Bass, and R. B. Merriman, "Health-related fitness and physical activity in patients with nonalcoholic fatty liver disease," Hepatology, vol. 47, no. 4, pp. 1158-1165, 2008.

[52] N. A. Johnson, T. Sachinwalla, D. W. Walton et al., "Aerobic exercise training reduces hepatic and visceral lipids in obese individuals without weight loss," Hepatology, vol. 50, no. 4, pp. 1105-1112, 2009.

[53] U. F. H. Leuschner, B. Lindenthal, G. Herrmann et al., "Highdose ursodeoxycholic acid therapy for nonalcoholic steatohepatitis: a double-blind, randomized, placebo-controlled trial," Hepatology, vol. 52, no. 2, pp. 472-479, 2010.

[54] M. K. Chae, S. G. Park, S.-O. Song et al., "Pentoxifylline attenuates methionine- and choline-deficient-diet-induced steatohepatitis by suppressing TNF- $\alpha$ expression and endoplasmic reticulum stress," Experimental Diabetes Research, vol. 2012, Article ID 762565, 8 pages, 2012.

[55] L. A. Adams, C. O. Zein, P. Angulo, and K. D. Lindor, "A pilot trial of pentoxifylline in nonalcoholic steatohepatitis," The American Journal of Gastroenterology, vol. 99, no. 12, pp. 23652368, 2004.

[56] A. J. Sanyal, N. Chalasani, K. V. Kowdley et al., "Pioglitazone, vitamin E, or placebo for nonalcoholic steatohepatitis," The New England Journal of Medicine, vol. 362, no. 18, pp. 1675-1685, 2010.

[57] V. Nobili, M. Manco, R. Devito et al., "Lifestyle intervention and antioxidant therapy in children with nonalcoholic fatty liver disease: a randomized, controlled trial," Hepatology, vol. 48, no. 1, pp. 119-128, 2008.

[58] J. E. Lavine, J. B. Schwimmer, M. L. Van Natta et al., "Nonalcoholic steatohepatitis clinical research network. Effect of vitamin E or metformin for treatment of nonalcoholic fatty liver disease in children and adolescents: the TONIC randomized controlled trial," Journal of the American Medical Association, vol. 305, no. 16, pp. 1659-1668, 2011.

[59] A. Hirose, M. Ono, T. Saibara et al., "Angiotensin II type 1 receptor blocker inhibits fibrosis in rat nonalcoholic steatohepatitis," Hepatology, vol. 45, no. 6, pp. 1375-1381, 2007.

[60] P. Riley, D. Sudarshi, M. Johal et al., "Weight loss, dietary advice and statin therapy in non-alcoholic fatty liver disease: 
a retrospective study," International Journal of Clinical Practice, vol. 62, no. 3, pp. 374-381, 2008.

[61] H. Park, T. Shima, K. Yamaguchi et al., "Efficacy of long-term ezetimibe therapy in patients with nonalcoholic fatty liver disease," Journal of Gastroenterology, vol. 46, no. 1, pp. 101-117, 2011.

[62] T. D. Filippatos and M. S. Elisaf, "Combination drug treatment in patients with non-alcoholic fatty liver disease," World Journal of Hepatology, vol. 2, no. 4, pp. 139-142, 2010.

[63] G. L. Vega, M. Chandalia, L. S. Szczepaniak, and S. M. Grundy, "Effects of N-3 fatty acids on hepatic triglyceride content in humans," Journal of Investigative Medicine, vol. 56, no. 5, pp. 780-785, 2008.

[64] L. Juurinen, M. Tiikkainen, A. M. Häkkinen, A. Hakkarainen, and H. Yki-Järvinen, "Effects of insulin therapy on liver fat content and hepatic insulin sensitivity in patients with type 2 diabetes," The American Journal of Physiology, vol. 292, no. 3, pp. E829-E835, 2007.

[65] N. A. Gupta, J. Mells, R. M. Dunham et al., "Glucagon-like peptide-1 receptor is present on human hepatocytes and has a direct role in decreasing hepatic steatosis in vitro by modulating elements of the insulin signaling pathway," Hepatology, vol. 51, no. 5, pp. 1584-1592, 2010.

[66] S. L. Samson and M. Bajaj, "Potential of incretin-based therapies for non-alcoholic fatty liver disease," Journal of Diabetes and Its Complications.

[67] V. G. Athyros, O. Giouleme, E. S. Ganotakis et al., "Safety and impact on cardiovascular events of long-term multifactorial treatment in patients with metabolic syndrome and abnormal liver function tests: a post hoc analysis of the randomised ATTEMPT study," Archives of Medical Science, vol. 7, no. 5, pp. 796-805, 2011.

[68] American Diabetes Association, "Clinical practice recommendations," Diabetes Care, vol. 26, Suppl 1, pp. S11-S67, 2013.

[69] E. Fassio, E. Álvarez, N. Domínguez, G. Landeira, and C. Longo, "Natural history of nonalcoholic steatohepatitis: a longitudinal study of repeat liver biopsies," Hepatology, vol. 40, no. 4, pp. 820-826, 2004.

[70] J. B. Dixon, P. S. Bhathal, and P. E. O'Brien, "Nonalcoholic fatty liver disease: predictors of nonalcoholic steatohepatitis and liver fibrosis in the severely obese," Gastroenterology, vol. 121, no. 1, pp. 91-100, 2001.

[71] D. L. White, F. Kanwal, and H. B. El-Serag, "Association between nonalcoholic fatty liver disease and risk for hepatocellular cancer, based on systematic review," Clinical Gastroenterology and Hepatology, vol. 10, no. 12, pp. 1342-1359, 2012.

[72] C. Söderberg, P. Stål, J. Askling et al., "Decreased survival of subjects with elevated liver function tests during a 28-year follow-up," Hepatology, vol. 51, no. 2, pp. 595-602, 2010.

[73] L. A. Adams, S. Harmsen, J. L. S. Sauver et al., "Nonalcoholic fatty liver disease increases risk of death among patients with diabetes: a community-based cohort study," The American Journal of Gastroenterology, vol. 105, no. 7, pp. 1567-1573, 2010. 


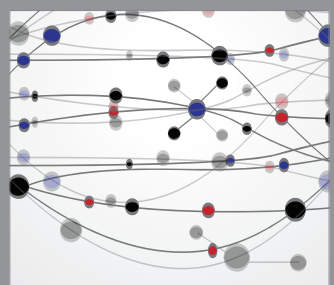

The Scientific World Journal
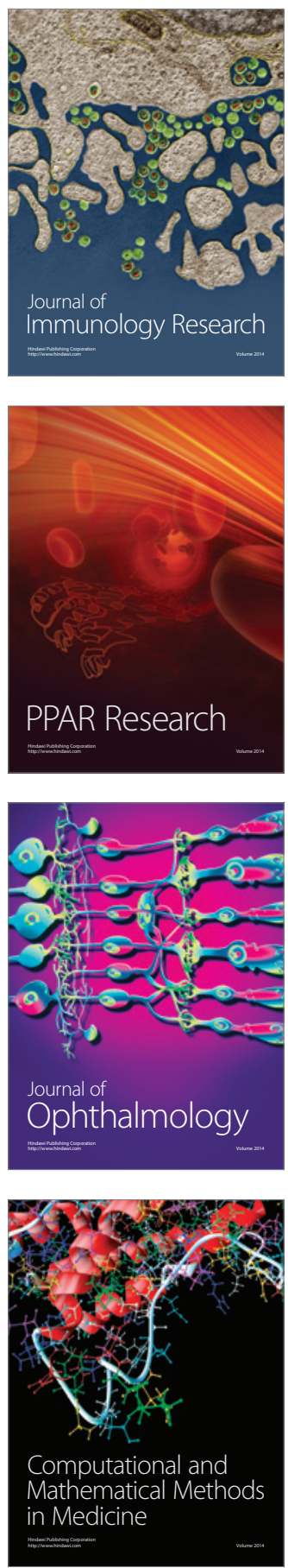

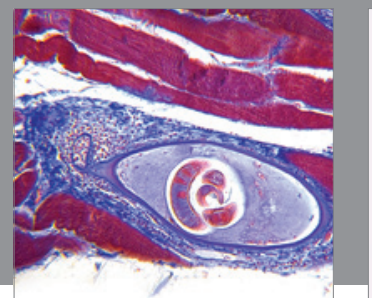

Gastroenterology

Research and Practice
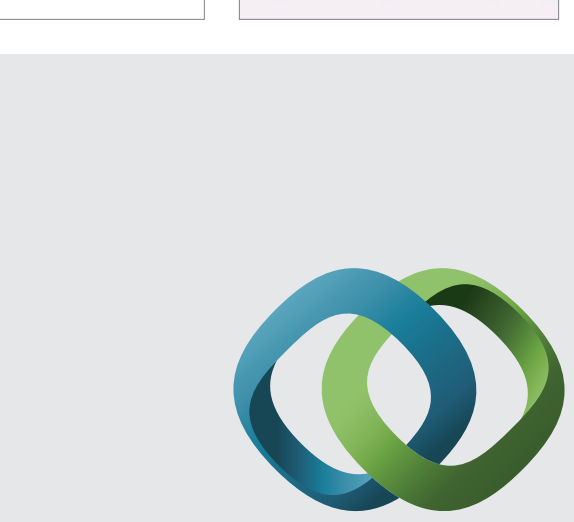

\section{Hindawi}

Submit your manuscripts at

http://www.hindawi.com
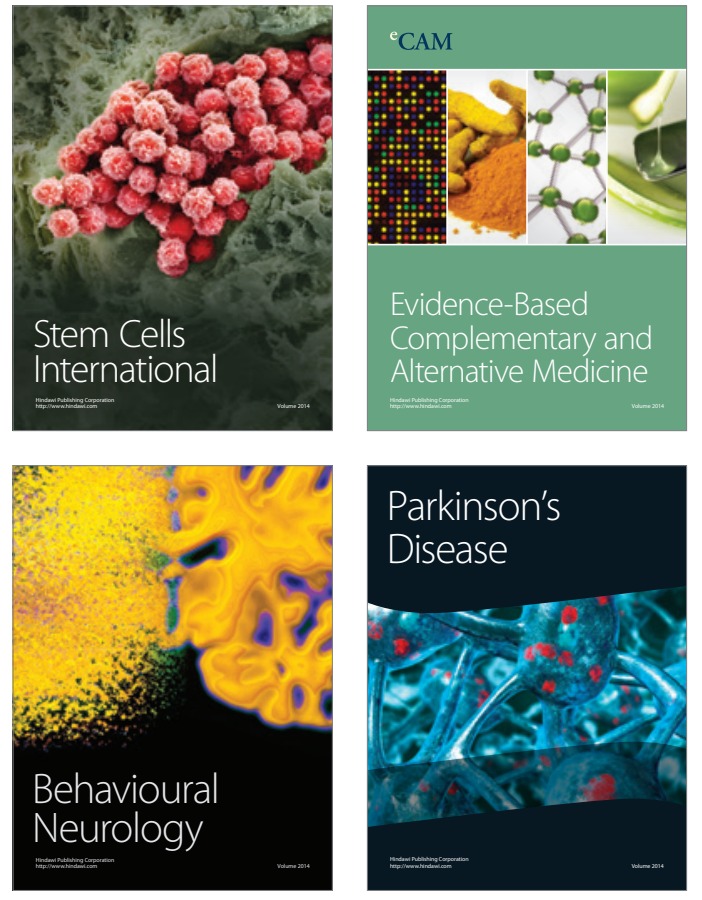
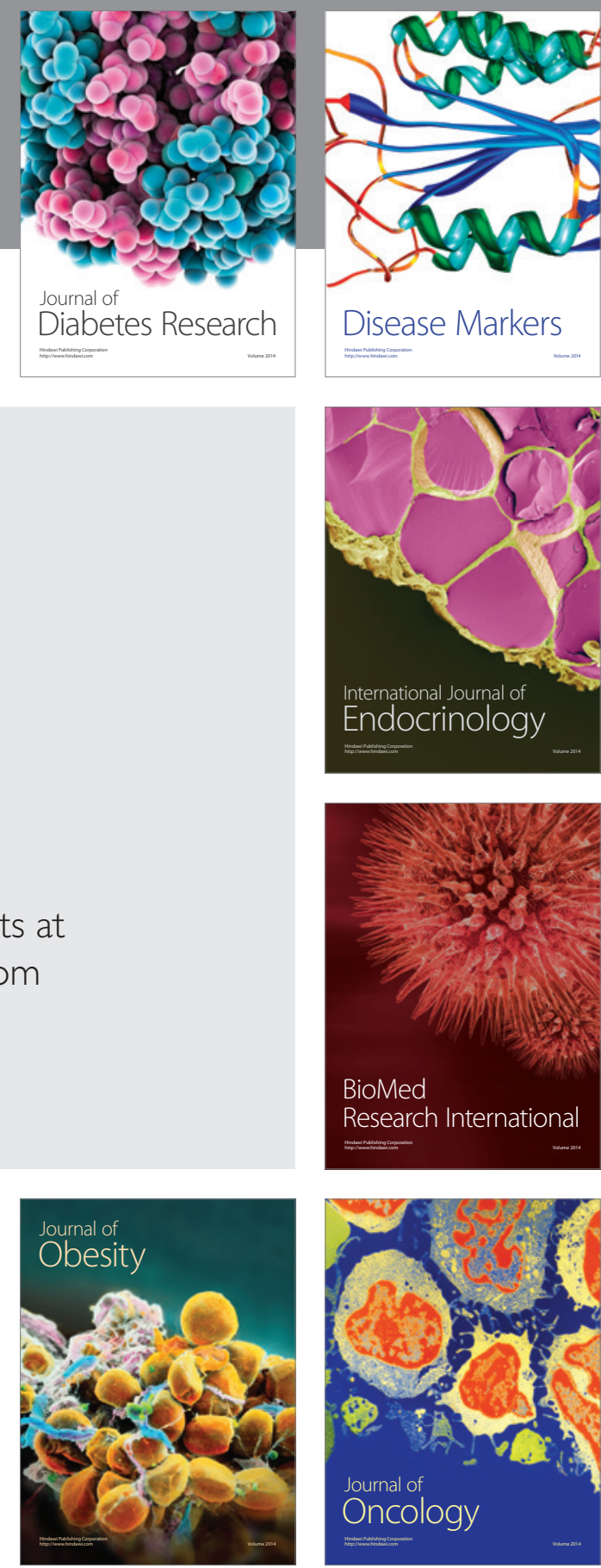

Disease Markers
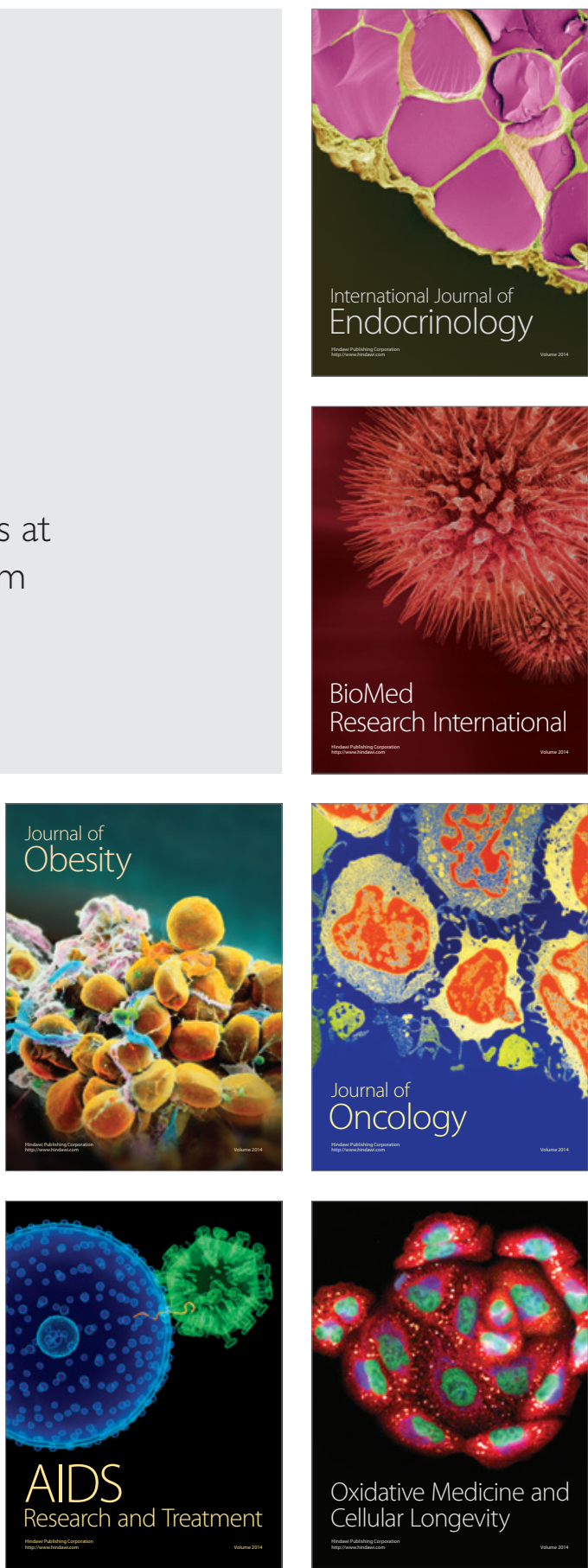